\title{
Mecamylamine selectively blocks nicotinic receptors on vasomotor sympathetic $\mathrm{C}$ neurons
}

\author{
Wei-Xing Shen, John P. Horn * \\ Department of Neurobiology, University of Pittsburgh School of Medicine, E1440 Biomedical Science Tower, Pittsburgh, PA 15261, USA
}

Accepted 9 December 1997

\begin{abstract}
Mecamylamine differentially blocked fast nicotinic transmission in two functional subsets of sympathetic neurons within lumbar paravertebral ganglia of the bullfrog. $\mathrm{EC}_{50} \mathrm{~s}$ for inhibition of postsynaptic compound action potentials were $27.3 \pm 2.5 \mu \mathrm{M}$ in the secretomotor B system and $5.7 \pm 0.7 \mu \mathrm{M}$ in the vasomotor $\mathrm{C}$ system. This $5.2: 1$ selectivity is 2.6 times greater than observed previously with D-tubocurarine, a nonselective blocker of nicotinic receptors, and it indicates that mecamylamine preferentially interacts with nicotinic receptors on sympathetic $\mathrm{C}$ neurons. We tested this by analyzing the effect of mecamylamine upon synaptic currents. In both cell types, the drug produced a qualitatively similar picture of open-channel blockade. It reduced EPSC amplitude, speeded EPSC decay, and became more effective with membrane hyperpolarization and repetitive activity. Despite these similarities, $8 \mu \mathrm{M}$ mecamylamine reduced EPSC amplitude to a greater extent in C neurons, and the rate constant for drug binding to open channels was 4.4 times greater in B cells, irrespective of membrane potential. This implies that the unblocking rate for mecamylamine is much slower in C cells than B cells, and it shows that the drug recognizes a structural difference between nicotinic receptors on these two populations of sympathetic neurons. (C) 1998 Elsevier Science B.V.
\end{abstract}

Keywords: Open-channel block; Neuronal nicotinic receptors; Bullfrog sympathetic ganglia; Blood pressure

\section{Introduction}

The goal of this study was to identify a drug that could distinguish between nicotinic receptors on different sympathetic neurons. We were motivated by emerging evidence that functional subsets of autonomic neurons may express distinct patterns of nicotinic receptor subunits. If true, then selective nicotinic antagonists could provide the means for manipulating different components of autonomic reflexes.

Neuronal nicotinic receptors are a diverse group of pentameric proteins formed by eleven genes which code for eight $\alpha$ and three $\beta$ subunits [26]. Although the postsynaptic receptors mediating fast nicotinic transmission in autonomic ganglia are generally thought to contain two $\alpha$ and three $\beta$ subunits, their exact subunit stoichiometry remains controversial. The problem arises because native expression of subunits occurs in a complex pattern. At least five different subunits $(\alpha 3, \alpha 5, \alpha 7, \beta 2$ and $\beta 4)$

\footnotetext{
* Corresponding author. Fax: + 1-412-648-1441; E-mail: jph+ @ pitt.edu
}

are always found in sympathetic and parasympathetic ganglia of the chicken and rat $[4,11,16,21,25]$. In some cases, additional subunits are also present; $\alpha 4$ in chicken and rat sympathetic ganglia [11,25], $\alpha 2, \alpha 4$ and $\beta 3$ in rat cardiac ganglion [21]. At the level of individual neurons, Poth et al. [21] found considerable heterogeneity of RNA transcripts for different nicotinic subunits in the mainly parasympathetic rat cardiac ganglion. They also observed a correlation between cellular patterns of nicotinic subunit expression and four types of receptors having distinct single channel conductances. This provided the first molecular evidence that subsets of autonomic neurons may express different nicotinic receptors, but was presaged by earlier studies of synaptic currents [17] and receptor expression.

Heterologous expression of cloned nicotinic subunits can produce receptors having distinct pharmacological profiles in addition to differences in single channel conductance and gating $[12,13,20,24]$. Interestingly, the properties of receptors assembled from pairwise expression of $\alpha$ and $\beta$ subunits in Xenopus oocytes do not fully match those of native receptors on autonomic neurons [5]. These findings 
lend support to the proposal that synaptic receptors on autonomic neurons normally assemble from three or more types of subunits $[11,29]$, and they provide the best available evidence that antagonists may be useful in distinguishing neuronal receptor subtypes.

Although nicotinic receptors are found on all autonomic ganglion cells, these neurons are phenotypically diverse. Beginning with the classical work of Langley [9], the autonomic nervous system has been conceptualized as having sympathetic, parasympathetic and enteric divisions based on structural and functional grounds. Contemporary efforts have delineated cellular specializations in each division. Within the sympathetics, evidence derived from mammals, birds, and amphibians supports the general principle that postganglionic neurons are heterogeneous in their phenotypic properties and functional roles. In the cat, e.g., as many as thirteen subsets of sympathetic neurons can be differentiated by their target specificity and cellular properties [7]. Specialized subsets of neurons selectively control different vascular beds, glands, and piloerector muscles, and they express differences in action potential conduction velocities and in synthesis profiles of transmitters and metabotropic receptors [19].

In the present work, we focused on bullfrog sympathetic ganglia, a system where the organization of neuronal phenotypes is relatively simple. Paravertebral ganglia 9 and 10 contain only two major cell groups: B neurons innervate cutaneous glands [8] and $\mathrm{C}$ neurons innervate arteries [28]. A difference in the kinetic properties of nicotinic receptors on $\mathrm{B}$ and $\mathrm{C}$ neurons is evident in synaptic currents and acetylcholine noise [17,27]. The time constant $(\tau)$ for exponential decay of the nicotinic EPSC is $\sim 5 \mathrm{~ms}$ in B cells and $\sim 10 \mathrm{~ms}$ in $\mathrm{C}$ cells. This implies a difference in the mean duration of individual channel openings or bursts of openings [18]. Nicotinic receptors on $\mathrm{B}$ and $\mathrm{C}$ cells must therefore differ in some aspect of their structure, possibly subunit composition. After screening several compounds with simple extracellular recording, we identified mecamylamine as a drug that appeared selective for the vasomotor $\mathrm{C}$ system. Here, we describe these initial experiments and present additional evidence from analysis of synaptic currents that mecamylamine binds with different affinity to receptors on secretomotor $\mathrm{B}$ neurons and vasomotor $\mathrm{C}$ neurons.

\section{Materials and methods}

Preparations of paravertebral ganglia 7-10 were dissected from adult bullfrogs (13-18 cm in length) obtained from Charles D. Sullivan (Nashville, TN). Another paper [27] details the methods for dissection, selective stimulation of the preganglionic $\mathrm{B}$ and $\mathrm{C}$ pathways, extracellular recording of postganglionic compound action potentials, 2-electrode voltage-clamp measurements of synaptic cur- rents, and for digitization of the data. Grouped data are expressed as the mean \pm SEM.

Mecamylamine $\mathrm{HCl}$ was obtained from Research Biochemicals (Natick, MA). In experiments using extracellular recording to measure dose-response curves, mecamylamine was added to normal Ringer composed of (mM) $115 \mathrm{NaCl}, 2 \mathrm{KCl}, 1.8 \mathrm{CaCl}_{2}$, 4 Na-HEPES (pH 7.2 - 7.3). In voltage-clamp experiments, the $\mathrm{CaCl}_{2}$ concentration was raised to $4 \mathrm{mM}$ in order to stabilize the recordings.

\subsection{Voltage-clamp protocol}

Synaptic currents were recorded from individual neurons over a range of three drug concentrations and four holding potentials. During the entire protocol, the presynaptic nerve was continuously stimulated at $0.1 \mathrm{~Hz}$. Measurements of EPSC amplitude and decay were made from averages of 3 responses. After recording control EPSCs in drug-free Ringer, $1 \mu \mathrm{M}$ mecamylamine was introduced at $3 \mathrm{ml} \mathrm{min}{ }^{-1}$ into the $1 \mathrm{ml}$ recording chamber and allowed to equilibrate for 8-10 min. A family of EPSCs was then recorded at holding potentials of $-50,-60,-80$, and $-100 \mathrm{mV}$, and the process was repeated for 3 and $8 \mu \mathrm{M}$ mecamylamine. Only cells that remained stable during the entire protocol $(45-60 \mathrm{~min})$ were included in the kinetic analysis.

\subsection{Analysis of synaptic currents}

A simple sequential kinetic model for open-channel block was used to analyze the effect of mecamylamine upon EPSC decay [22,23]. The model and the associated reaction rates are shown below ( $A$ refers to acetylcholine, $R$ to the nicotinic receptor, $B$ to the blocking drug, and $\left[X_{\mathrm{B}}\right]$ to its concentration.).

$$
\text { closed } \begin{gathered}
\text { binding } \\
k_{+1}
\end{gathered} \underset{\mathrm{A}}{\underset{\substack{k_{-1} \\
\text { unbinding }}}{\rightleftharpoons}} \mathrm{AR} \underset{\substack{\alpha \\
\text { closing }}}{\stackrel{\text { opening }}{\beta}} \mathrm{AR}^{*}+\mathrm{B} \underset{\substack{k_{-\mathrm{B}} \\
\text { unblocking }}}{\rightleftharpoons} \mathrm{AR}^{*} \mathrm{~B} .
$$

If one assumes that the lifetime of acetylcholine in the synaptic cleft is brief, and that the agonist binding and unbinding reaction rates $\left(k_{+1}, k_{-1}\right)$ are much faster than channel gating $(\alpha, \beta)[14,15]$, then the time constant $(\tau)$ for EPSC decay is equal to $1 / \alpha$, where $\alpha$ is the rate of channel closing. This model predicts that an open-channel blocker should speed EPSC decay because the blocking reaction provides a second pathway for leaving the open state beyond normal channel closing. Under conditions where $k_{+\mathrm{B}}^{*} \gg k_{-\mathrm{B}}^{*}$, the apparent rate constant for EPSC decay $\left(\alpha^{\prime}\right)$ will equal the sum of two rates for leaving the open state $\left(\alpha^{\prime}=k_{+\mathrm{B}}^{*}\left[X_{\mathrm{B}}\right]+\alpha\right)$ [23]. We measured $\alpha$ and $\alpha^{\prime}$ from the reciprocal of time constants for EPSC decay in control and drug-containing solutions and then estimated $k_{+\mathrm{B}}^{*}$ from the slope of the linear relation between $\left[X_{\mathrm{B}}\right]$ and $\alpha^{\prime}$. 
A

Control

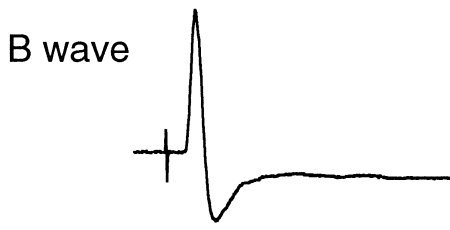

C wave

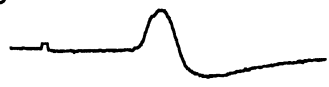

$20 \mu \mathrm{M}$ Mecamylamine

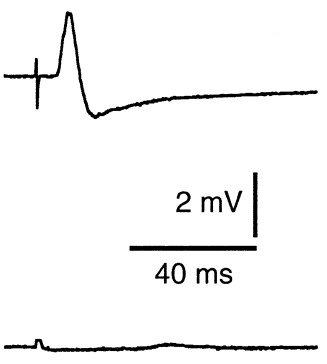

$B$

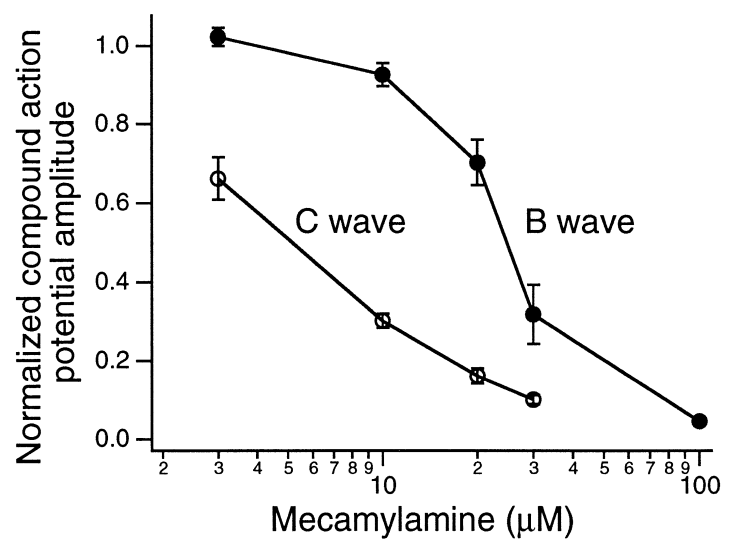

Fig. 1. Mecamylamine selectively antagonizes synaptic transmission in the vasomotor C pathway. (A) Extracellular compound action potentials were recorded from a ramus communicantes and were evoked by selectively stimulating the preganglionic $\mathrm{B}$ and $\mathrm{C}$ pathways through separate electrodes. The resulting $\mathrm{B}$ and $\mathrm{C}$ waves had different latencies due to their different conduction velocities. Addition of $20 \mu \mathrm{M}$ mecamylamine caused more complete block of the $\mathrm{C}$ wave. (B) Cumulative dose-response curves contain data from 6 experiments. Compound action potential amplitudes in each experiment were normalized to pre-drug controls and then averaged.

\section{Results}

Mecamylamine selectively blocked fast synaptic transmission in the vasomotor $\mathrm{C}$ system. Prior to drug addition, postganglionic compound action potentials remained stable when either the preganglionic B or C pathway was selectively stimulated at $0.1 \mathrm{~Hz}$. Fig. $1 \mathrm{~A}$ illustrates one experiment in which $20 \mu \mathrm{M}$ mecamylamine produced only partial block of the postsynaptic B wave, but nearly full block of the $\mathrm{C}$ wave. Cumulative dose-response curves compiled from 6 such experiments (Fig. 1B) showed that the $\mathrm{EC}_{50}$ for mecamylamine was $27.3 \pm 2.5 \mu \mathrm{M}$ in the $\mathrm{B}$ pathway and $5.7 \pm 0.7 \mu \mathrm{M}$ in the $\mathrm{C}$ pathway. The selectivity ratio of $\mathrm{EC}_{50} \mathrm{~s}$ in the $\mathrm{B}$ and $\mathrm{C}$ paths was therefore 5.2:1. By comparison, D-tubocurarine has a selectivity ratio of only 2.1:1 when measured in this system using the same

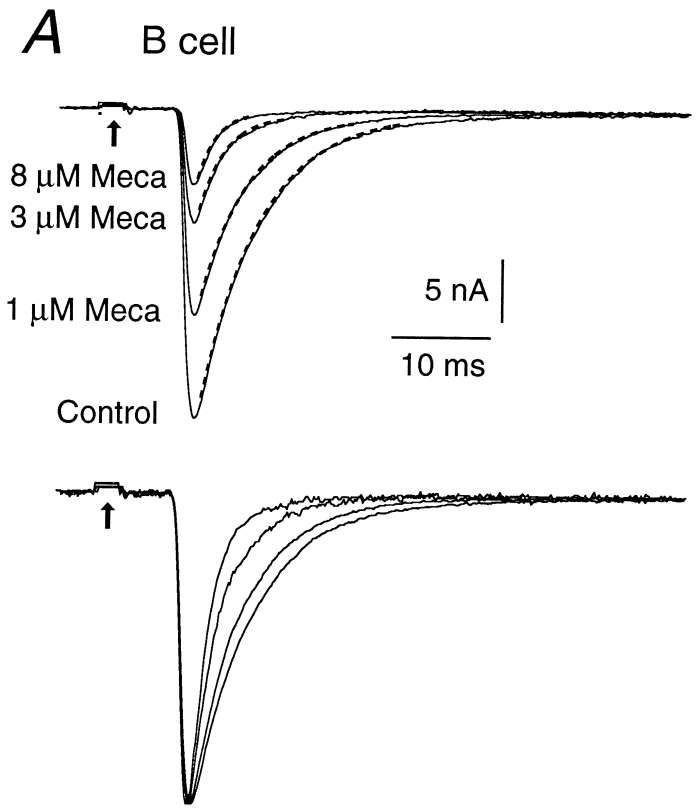

B $\mathrm{c}$ cell
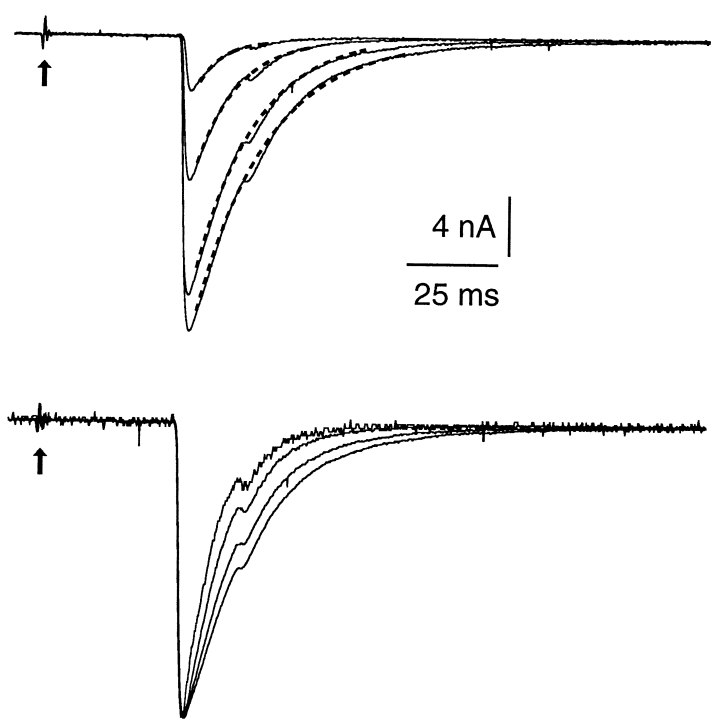

Fig. 2. Mecamylamine inhibits synaptic currents. Families of synaptic currents were recorded from a B neuron (A) and a C neuron (B) in drug-free Ringer and 3 doses of mecamylamine. In the upper panels, the dashed lines superimposed on the decay phase of EPSCs are single-exponential fits of the data. The small notch on the decay of the $\mathrm{C}$ cell was due to a secondary nicotinic synapse present on this cell. In the lower panels, all EPSCs have been scaled to one amplitude. EPSC decay was slowest in drug-free Ringer and became progressively faster with increasing concentrations of mecamylamine. 


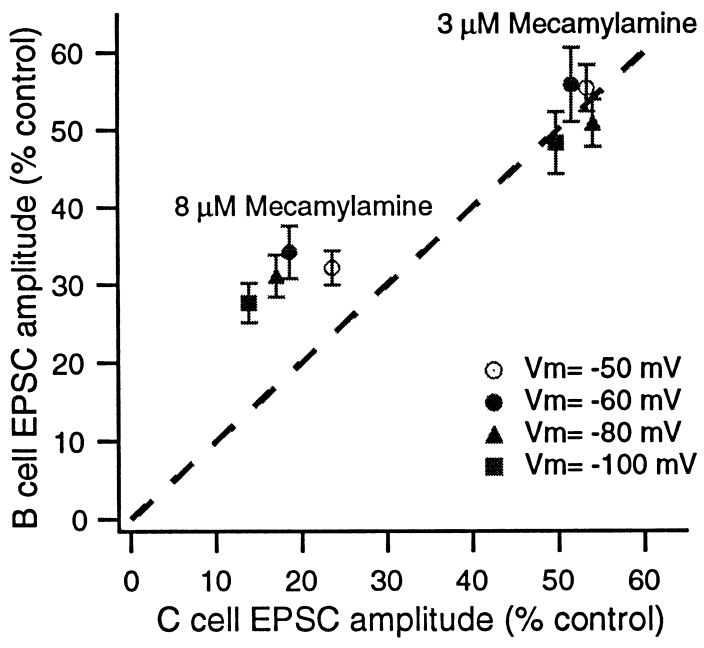

Fig. 3. Cell-specific inhibition of synaptic currents. EPSC amplitudes (6 $\mathrm{B}$ neurons and $2 \mathrm{C}$ neurons) recorded in 2 doses of mecamylamine were normalized to their pre-drug control values. Averaged data were then plotted for B vs. C cells in order to identify cell selectivity in the form of deviations from the diagonal (dashed line). Selectivity for receptors on $\mathrm{C}$ neurons was apparent at $8 \mu \mathrm{M}$, but not $3 \mu \mathrm{M}$ mecamylamine.

method [27]. In bullfrog sympathetic ganglia, D-tubocurarine acts as a competitive antagonist of nicotinic receptors $[10,27]$. Selectivity for synapses on $\mathrm{C}$ neurons does not arise from a cell-specific difference in drug affinity, but rather from the fact that the nicotinic EPSP in B cells has a larger safety factor for exceeding action potential threshold [27]. Since the safety factor is independent of the drug used to block receptors, the larger selectivity observed with mecamylamine shows that it must act through a different mechanism than D-tubocurarine. We tested this hypothesis by recording synaptic currents.

In Ringer containing $4.0 \mathrm{mM} \mathrm{Ca}^{2+}$, peak EPSC amplitude at $-50 \mathrm{mV}$ was $14.3 \pm 4.6 \mathrm{nA}(n=6)$ in $\mathrm{B}$ neurons and $12.1 \pm 4.0 \mathrm{nA}(n=6)$ in $\mathrm{C}$ neurons. In both cell types, mecamylamine (1-8 $\mu \mathrm{M})$ inhibited EPSC amplitude and speeded EPSC decay (Fig. 2). The increases in decay rates are most easily seen after normalizing EPSCs recorded in different drug concentrations to the same amplitude (Fig. 2 , bottom traces).

The selectivity of mecamylamine for nicotinic transmission in the $\mathrm{C}$ pathway suggests that it might preferentially block EPSC amplitude in C neurons. This was the case for $8 \mu \mathrm{M}$ mecamylamine, but not for lower doses. Fig. 3 compares the block of ESPC amplitude in B $(n=6)$ vs. C $(n=2)$ neurons at two drug concentrations. In such a plot, selectivity for receptors on one cell type will result in deviation from the diagonal. All data for $8 \mu \mathrm{M}$ mecamylamine were to the left of the diagonal, thus indicating a preferential block of receptors on $\mathrm{C}$ cells. This effect became slightly larger when the membrane potential was increased from -50 to $-100 \mathrm{mV}$. By contrast, $3 \mu \mathrm{M}$ mecamylamine was equally effective for receptors on the two cell types.

The shortening of EPSC decay times in mecamylamine indicates it inhibits synaptic transmission by blocking the open nicotinic channel. Before and after exposure to
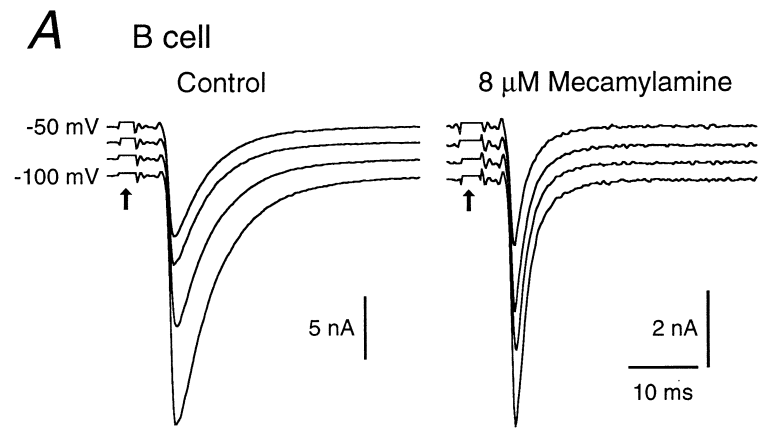

$B$

C
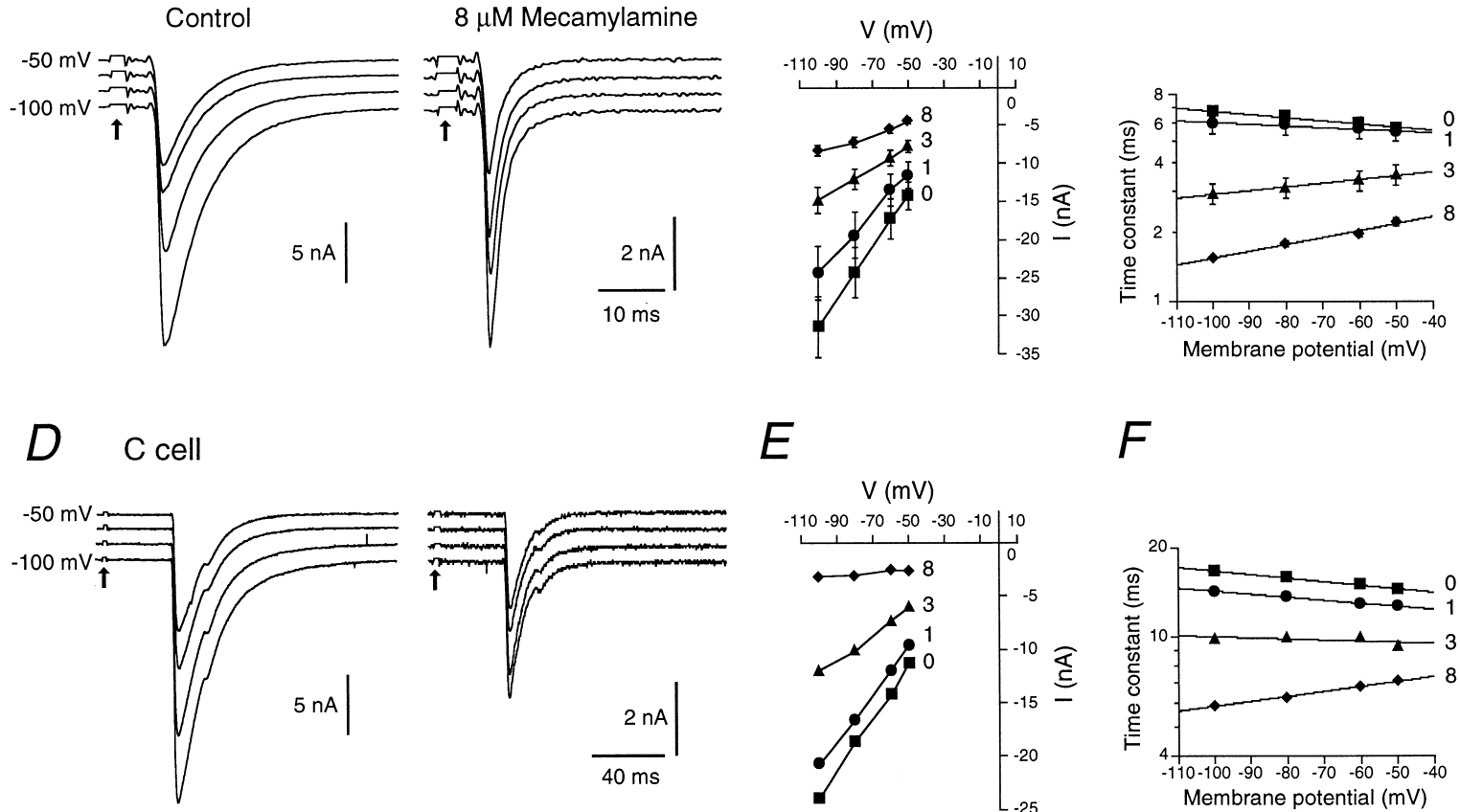

E

$F$
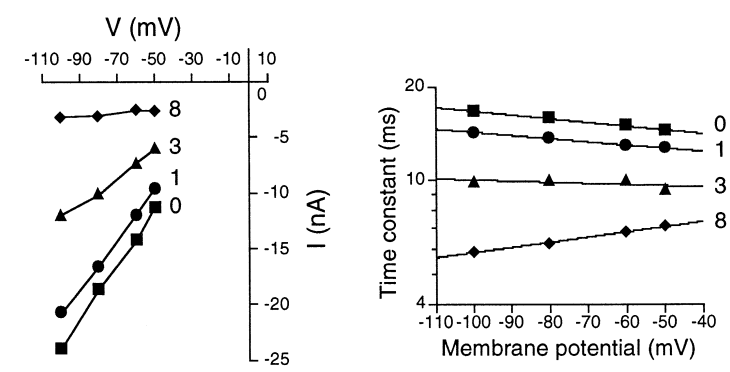

Fig. 4. The block of synaptic currents is voltage-dependent. The effect of $8 \mu \mathrm{M}$ mecamylamine at four holding potentials is shown for a B neuron (A) and a C neuron (D). Panels B and E plot I-V relations of grouped EPSC data from six B and two C cells. Numbers at the right of each curve denote the dose of mecamylamine $(\mu \mathrm{M})$. Panels $\mathrm{C}$ and $\mathrm{F}$ show the time constant of EPSC decay varied with membrane potential and mecamylamine concentration. 


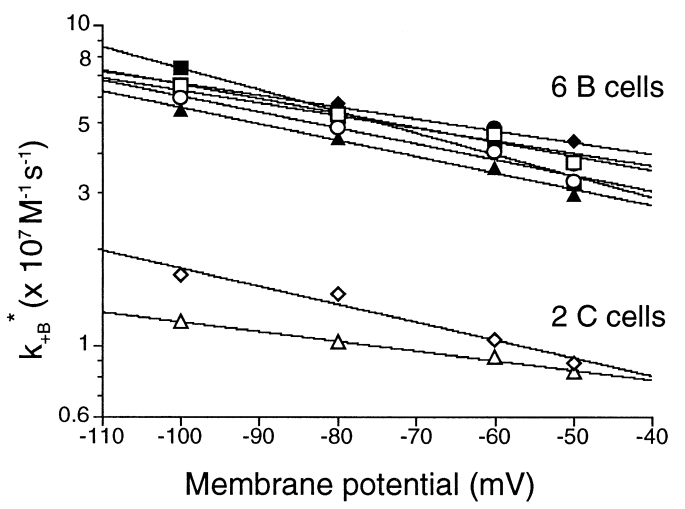

Fig. 5. $k_{+\mathrm{B}}^{*}$, the binding constant for mecamylamine block, has a similar exponential voltage-dependence in $\mathrm{B}$ and $\mathrm{C}$ neurons, but differs in magnitude by a factor of 4.4 between the two cell types.

mecamylamine, EPSCs in both cell types were best fit by a single exponential function rather than as the sum of two exponentials (Fig. 2). This implies that the unblocking rate for mecamylamine is much slower than the time course of the EPSC, and permits use of a simple method for estimating the binding rate constant of mecamylamine [23].

Since mecamylamine is a monovalent cation at physiological $\mathrm{pH}$, its ability to bind to a site within the open nicotinic channel should increase with membrane hyperpolarization, if the site lies within the plane of the membrane. This was indeed the case, as can be seen from representative families of EPSCs recorded at four holding potentials in a B cell (Fig. 4A) and a C cell (Fig. 4D). In agreement with previous work, EPSC amplitude increased linearly in drug-free Ringer as the holding potential was shifted from -50 to $-100 \mathrm{mV}$ [27]. Hyperpolarization of this magnitude causes an approximate doubling of the driving force on synaptic currents which normally reverse near $0 \mathrm{mV}$ in both cell types. In records from individual neurons (Fig. 4A,D) and I-V plots of grouped data (Fig. $4 \mathrm{~B}, \mathrm{E})$, one can see that $8 \mu \mathrm{M}$ mecamylamine caused modest outward rectification of the $\mathrm{I}-\mathrm{V}$ relation in six $\mathrm{B}$ cells (Fig. 4B) and a much stronger effect in two C cells (Fig. 4E). Rectification of this kind is a hallmark of open-channel occlusion which tends to increase as membrane polarization drives the drug into channels.

The voltage-dependence of mecamylamine block was also evident in time constants $(\tau)$ of EPSC decay. Normally, in drug-free Ringer, $\tau$ itself is voltage-dependent and increases with membrane hyperpolarization in both cell types [17,27]. Mecamylamine shifted this relation to the point where, at higher doses, $\tau$ decreased instead with hyperpolarization (Fig. 4C,F). The data also show that at each holding potential, $\tau$ decreased as the dose of mecamylamine was increased.

Using a sequential blocking scheme for channel kinetics, we estimated $k_{+\mathrm{B}}^{*}$, the binding constant for mecamylamine to open channels, from the slope of the relation between drug concentration and the reciprocal time constant for EPSC decay (Methods and materials). Comparing the data from individual neurons (Fig. 5) shows that $k_{+\mathrm{B}}^{*}$ was 4.4 fold greater in B neurons, but it has a similar voltage-dependence in both cell types. The binding constant $k_{+\mathrm{B}}^{*}$ always increased exponentially with membrane hyperpolarization (Fig. 5) and could be described by the equation:

$k_{+\mathrm{B}}^{*}(V)=k_{+\mathrm{B}}^{*}(0) \exp \left[V\left(H_{+\mathrm{B}}^{*}\right)^{-1}\right]$,

where $k_{+\mathrm{B}}^{*}$ is the rate constant at potentials of $\mathrm{V}$ and 0 $\mathrm{mV}$, and $H_{+\mathrm{B}}^{*}$ is the shift in membrane potential that produces an e-fold increase of $k_{+\mathrm{B}}^{*}$. Grouping the results showed that $k_{+\mathrm{B}}^{*}(0)=2.15 \pm 0.20 \times 10^{7} \mathrm{M}^{-1} \mathrm{~s}^{-1}$ and
$A$

Control
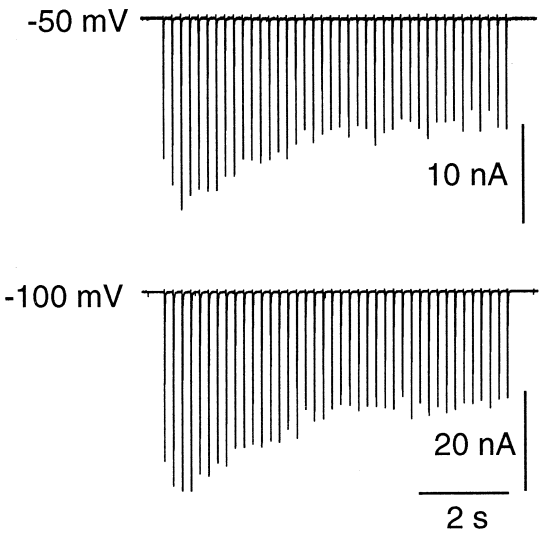

$3 \mu \mathrm{M}$ Mecamylamine
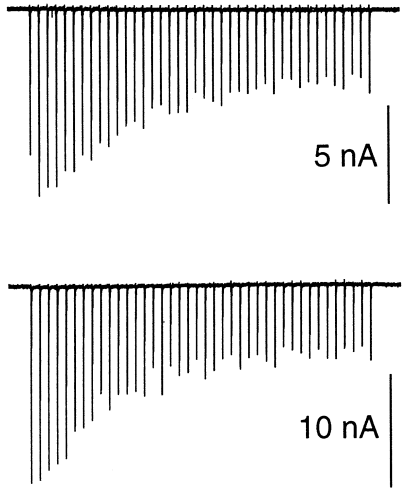

$B$

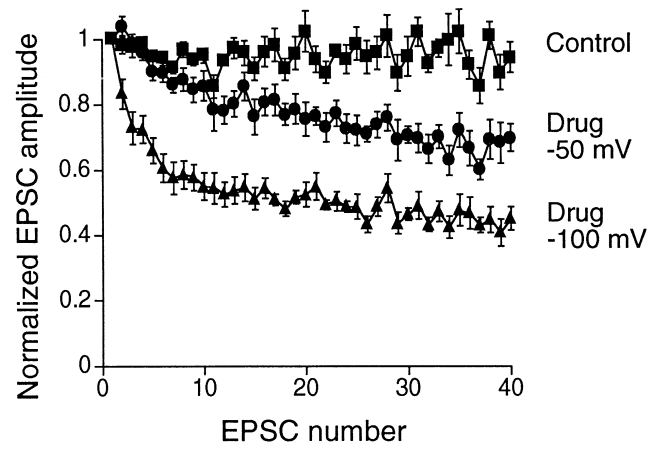

Fig. 6. The action of mecamylamine is use-dependent and increases with hyperpolarization. Panel A illustrates trains of 40 EPSCs evoked at $5 \mathrm{~Hz}$ in a B neuron. Panel B shows grouped data from six cells. In each train, EPSC amplitudes were initially normalized to the first response in the train. The data points labeled control represent normalized EPSCs recorded at $-100 \mathrm{mV}$ divided by corresponding EPSCs recorded at $-50 \mathrm{mV}$. The other two data sets show the effect of $3 \mu \mathrm{M}$ mecamylamine upon trains after normalization to control EPSCs at $-50 \mathrm{mV}$. 
$H_{+\mathrm{B}}^{*}=-94 \pm 9 \mathrm{mV}$ in six B cells and that $k_{+\mathrm{B}}^{*}(0)=0.54$ $\times 10^{7} \mathrm{M}^{-1} \mathrm{~s}^{-1}$ and $H_{+\mathrm{B}}^{*}=-111 \mathrm{mV}$ in two $\mathrm{C}$ cells. Using a Woodhull-type model for channel block by an ion [1], we converted $H_{+\mathrm{B}}^{*}$ to $\delta$, the electrical distance across the membrane field where binding occurs, and found it was 0.28 in B cells and 0.25 in C cells.

During experiments on $\mathrm{B}$ neurons, we also examined the use-dependence of mecamylamine block. The possibility that the unblocking rate is slow relative to $k_{+\mathrm{B}}^{*}$ is suggested by the lack of a slow component of EPSC decay. A slow unblocking rate could lead to accumulation of mecamylamine block during repetitive synaptic activation. We tested this by recording trains of 40 EPSCs at 5 $\mathrm{Hz}$ (Fig. 6A). In drug-free Ringer, EPSC amplitude was characterized by transient facilitation followed by depression. Increasing the holding potential from $-50 \mathrm{mV}$ to $-100 \mathrm{mV}$ increased the magnitude of currents without changing the normal progression of facilitation and depression. Consequently, the ratio of normalized EPSC amplitudes recorded at the two potentials remained flat in the absence of the drug (Fig. 6B). By contrast, $3 \mu \mathrm{M}$ mecamylamine caused the depressed phase of EPSC amplitudes to become more pronounced at both potentials (Fig. 5A). Normalizing the profiles of EPSC amplitudes to drug-free controls showed clear use-dependent accumulation of EPSC blockade at $-50 \mathrm{mV}$ (Fig. 6B). Hyperpolarization to $-100 \mathrm{mV}$ increased the rate of onset rate and the magnitude of use-dependent block by mecamylamine.

\section{Discussion}

We have shown that mecamylamine selectively blocks synaptic transmission in the vasomotor sympathetic C system of the bullfrog, and that it can distinguish between nicotinic receptors on $\mathrm{B}$ and $\mathrm{C}$ neurons. In both cell types, mecamylamine acts as an open-channel blocker whose efficacy increases with hyperpolarization. At all potentials tested, an $8 \mu \mathrm{M}$ dose of mecamylamine was more effective in reducing EPSC amplitude in C neurons. Interestingly our estimates of $k_{+\mathrm{B}}^{*}$ show that the drug binds with higher affinity to receptors on B neurons. Given the overall selectivity for $\mathrm{C}$ cells this implies that the unblocking rate of mecamylamine must be slower in $\mathrm{C}$ than in $\mathrm{B}$ neurons.

Could the differential effect of mecamylamine upon synaptic transmission in B and C cells arise from a systematic difference in resting potential? Although difficult to rule out, this possibility seems unlikely. Available reports imply $\mathrm{B}$ and $\mathrm{C}$ neurons have similar resting potentials in the range of $-50--55 \mathrm{mV}$ [e.g., Refs. [10,17,27]]. In any event, the voltage-clamp data show how mecamylamine can distinguish between receptors on the two cell types.

Our voltage-clamp experiments were done in $4.0 \mathrm{mM}$ calcium to increase the stability of the recordings. When compared with previous measurements in $1.8 \mathrm{mM}$ calcium [27], raising extracellular calcium increased the synaptic conductance and $\tau$ in both cell types. We did not study the underlying mechanism for these changes but they probably arise from at least three factors; enhanced acetylcholine release, increased calcium current through open nicotinic channels, and increased probability that nicotinic channels open in response to acetylcholine [2].

Mecamylamine has previously been studied in parasympathetic neurons of the rat submandibular ganglion $[3,6]$. At low concentrations, it has no effect upon channel kinetics and behaves as a competitive blocker of acetylcholine binding to nicotinic receptors. Our finding that drug concentrations $\leq 3 \mu \mathrm{M}$ are equally effective in $\mathrm{B}$ and $\mathrm{C}$ cells in blocking EPSC amplitude raises the possibility that another mechanism, possibly competitive, operates in conjunction with open-channel block. At the very least, it shows that a simple open-channel blocking scheme with only one blocked state cannot fully describe mecamylamine's mechanism of action. Interestingly, Ascher et al. [3] noted in one of their studies a subset of parasympathetic neurons in which mecamylamine also produced a voltage-dependent block of nicotinic currents. At this time, we cannot account for the differences in mecamylamine action between frog and rat ganglia or within them. Does the range of results reflect fundamental differences between functional groups of autonomic neurons, between amphibians and mammals, or both? This question highlights the basic gaps in available molecular information. The identities of neuronal nicotinic receptor subunits are completely unknown in amphibians, and subunit expression has not been studied in the rat submandibular ganglion. One would expect that fundamental aspects of receptor structure and function are highly conserved based on available comparative data describing muscle and neuronal nicotinic receptors in different species [26]. Future cloning of amphibian neuronal nicotinic receptor subunits could provide structural insights into mecamylamine binding and into receptor function in specialized subsets of autonomic neurons.

\section{Acknowledgements}

This work was supported by a grant-in-aid from the American Heart Association, Pennsylvania Affiliate.

\section{References}

[1] D.J. Aidley, P.R. Stanfield, Ion Channels, Cambridge Univ. Press, Cambridge, England, 1996.

[2] M. Amador, J.A. Dani, Mechanism for modulation of nicotinic acetylcholine receptors that can influence synaptic transmission, J. Neurosci. 15 (1995) 4525-4532.

[3] P. Ascher, W.A. Large, H.P. Rang, Studies on the mechanism of action of acetylcholine antagonists on rat parasympathetic ganglion cells, J. Physiol. 295 (1979) 139-170. 
[4] R.A. Corriveau, D.K. Berg, Coexpression of multiple acetylcholine receptor genes in neurons: quantification of transcripts during development, J. Neurosci. 13 (1993) 2662-2671.

[5] P.J.O. Covernton, H. Kojima, L.G. Sivilotti, A.J. Gibb, D. Colquhoun, Comparison of neuronal nicotinic receptors in rat sympathetic neurons with subunit paris expressed in Xenopus oocytes, J. Physiol. 481 (1994) 27-34.

[6] A.M. Gurney, H.P. Rang, The channel-blocking action of methonium compounds on rat submandibular ganglion cells, Br. J. Pharmacol. 82 (1984) 623-642.

[7] W. Janig, E.M. McLachlan, Characteristics of function-specific pathways in the sympathetic nervous system, Trends Neurosci. 15 (1992) 475-481.

[8] P. Jobling, In vitro relation between preganglionic sympathetic stimulation and activity of cutaneous glands in the bullfrog, J. Physiol. 494 (1996) 287-296.

[9] J.N. Langley, The Autonomic Nervous System, W. Heffer, Cambridge, England, 1921.

[10] D. Lipscombe, H.P. Rang, Nicotinic receptors of frog ganglia resemble pharmacologically those of skeletal muscle, J. Neurosci. 8 (1988) 3258-3265.

[11] M. Listerud, A.B. Brussaard, P. Devay, D.R. Colman, L.W. Role, Functional contribution of neuronal AChR subunits revealed by antisense oligonucleotides, Science 254 (1991) 1518-1521.

[12] C.W. Luetje, J. Patrick, Both $\alpha$ and $\beta$-subunit contribute to the agonist sensitivity of neuronal nicotinic acetylcholine receptors, J. Neurosci. 11 (1991) 837-845.

[13] C.W. Luetje, K. Wada, S. Rogers, S.N. Abramson, K. Tsuji, S. Heinemann, J. Patrick, Neurotoxins distinguish between different neuronal acetylcholine receptor subunit combinations, J. Neurochem. 55 (1990) 632-640.

[14] K.L. Magleby, C.F. Stevens, The effect of voltage on the time course of end-plate currents, J. Physiol. 223 (1972) 151-171.

[15] K.L. Magleby, C.F. Stevens, A quantitative description of end-plate currents, J. Physiol. 223 (1972) 173-197.

[16] A. Mandelzys, P.D. Koninck, E. Cooper, Agonist and toxin sensitivities of ACh-evoked currents on neurons expressing multiple nicotinic ACH receptor subunits, J. Neurophysiol. 74 (1995) 1212-1221.

[17] L.M. Marshall, Different synaptic channel kinetics in sympathetic B and C neurons of the bullfrog, J. Neurosci. 6 (1986) 590-593.
[18] A. Mathie, S.G. Cull-Candy, D. Colquhoun, Conductance and kinetic properties of single nicotinic acetylcholine receptor channels in rat sympathetic neurones, J. Physiol. 439 (1991) 717-750.

[19] J.L. Morris, I.L. Gibbins, Cotransmission and neuromodulation. In: G. Burnstock, C.H.V. Hoyle (Eds.), Autonomic neuroeffector mechanisms, Harwood, Chur, 1992, pp. 33-119.

[20] R.L. Papke, The kinetic properties of neuronal nicotinic receptors: general basis of functional diversity, Prog. Neurobiol. 41 (1993) 509-531.

[21] K. Poth, T.J. Nutter, J. Cuevas, M.J. Parker, D.J. Adams, C.W. Luetje, Heterogeneity of nicotinic receptor class and subunit mRNA expression among individual parasympathetic neurons from rat intracardiac ganglia, J. Neurosci. 17 (1997) 586-596.

[22] H.P. Rang, The characteristics of synaptic currents and responses to acetylcholine of rat submandibular ganglion cells, J. Physiol. 311 (1981) 23-55.

[23] H.P. Rang, The action of ganglionic blocking drugs on the synaptic responses of rat submandibular ganglion cells, Br. J. Pharmacol. 75 (1982) $151-168$.

[24] L.W. Role, Diversity in primary structure and function of neuronal nicotinic acetylcholine receptor channels, Curr. Opin. Neurobiol. 2 (1992) 254-262.

[25] G. Rust, J.-M. Burgunder, T.E. Lauterburg, A.B. Cachelin, Expression of neuronal nicotinic acetylcholine receptor subunit genes in the rat autonomic nervous system, Eur. J. Neurosci. 6 (1994) 478-485.

[26] P.B. Sargent, The diversity of neuronal nicotinic acetylcholine receptors, Ann. Rev. Neurosci. 16 (1993) 403-443.

[27] W.-X. Shen, J.P. Horn, A presynaptic mechanism accounts for the differential block of nicotinic synapses on sympathetic B and C neurons by D-tubocurarine, J. Neurosci. 15 (1995) 5025-5035.

[28] R. Thorne, J.P. Horn, Role of ganglionic cotransmission in sympathetic control of the isolated bullfrog aorta, J. Physiol. 498 (1997) 201-214.

[29] A.B. Vernallis, W.G. Conroy, D.K. Berg, Neurons assemble acetylcholine receptors with as many as three kinds of subunits while maintaining subunit segregation among receptor subtypes, Neuron 10 (1993) 451-464. 